\title{
Three-Stage Evolution from Nonscalable to Scalable Optical Properties of Thiolate-Protected Gold Nanoclusters
}

Meng Zhou, ${ }^{\dagger} \|$ Tatsuya Higaki, ${ }^{\dagger}, \|$ Yingwei Li, ${ }^{\dagger} \|$ Chenjie Zeng, ${ }^{\dagger}$ Qi Li, ${ }^{\dagger}$ Matthew Y. Sfeir, ${ }^{\dagger}$,

$$
\text { Rongchao Jin }{ }^{*} \dagger
$$

${ }^{\dagger}$ Department of Chemistry, Carnegie Mellon University, Pittsburgh, PA 15213, USA

${ }^{\star}$ Photonics Initiative, Advanced Science Research Center, City University of New York, New York, NY 10031, USA

${ }^{\S}$ Department of Physics, Graduate Center, City University of New York, New York, NY 10016, USA.

*To whom correspondence should be addressed. Email: rongchao@andrew.cmu.edu.

\|These authors contributed equally.

S1. Carrier dynamics of small sized gold nanoclusters

S2. Nanosecond transient absorption measurements on the $\mathrm{Au}_{38}$ nanocluster.

S3. Measurement on the band gap of the Aug9 nanocluster.

S4. Carrier dynamics of medium sized gold nanoclusters.

S5. Nanosecond transient absorption measurements on the $\mathrm{Au}_{64}$ nanocluster.

S6. Decay associated spectra obtained from global fitting of $\mathrm{Au}_{64}, \mathrm{Au}_{92}$ and $\mathrm{Au}_{99}$ nanoclusters.

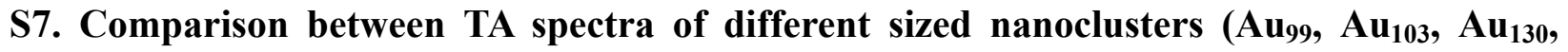
$\left.\mathrm{Au}_{133}, \mathbf{A u} \mathbf{u}_{144}\right)$

S8. Coherent oscillations observed in large-sized gold nanoclusters $\left(\mathrm{Au}_{99}, \mathbf{A u}_{103}, \mathbf{A u}_{130}, \mathbf{A u}_{133}\right.$, $\left.\mathrm{Au}_{144}, \mathrm{Au}_{246}\right)$ 
S1. Carrier dynamics of small sized gold nanoclusters
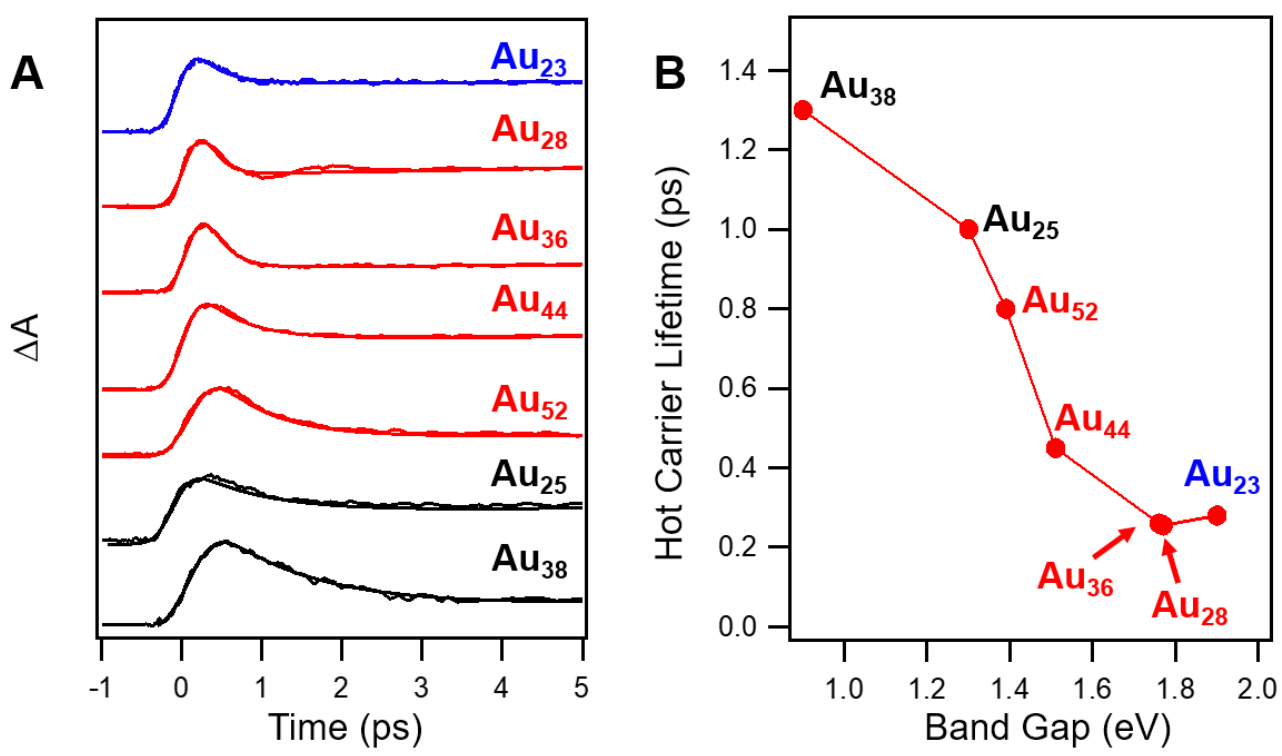

Figure S1. (A) TA decay traces at selected wavelengths of different sized gold nanoclusters between -1 and 5 ps. (B) Hot carrier lifetimes (picosecond) as a function of band gap of gold nanoclusters. It can be found that as $E_{\mathrm{g}}$ increases, the hot carrier cooling becomes faster. 
S2. Nanosecond transient absorption measurements on $\mathrm{Au}_{38}$ nanoclusters.
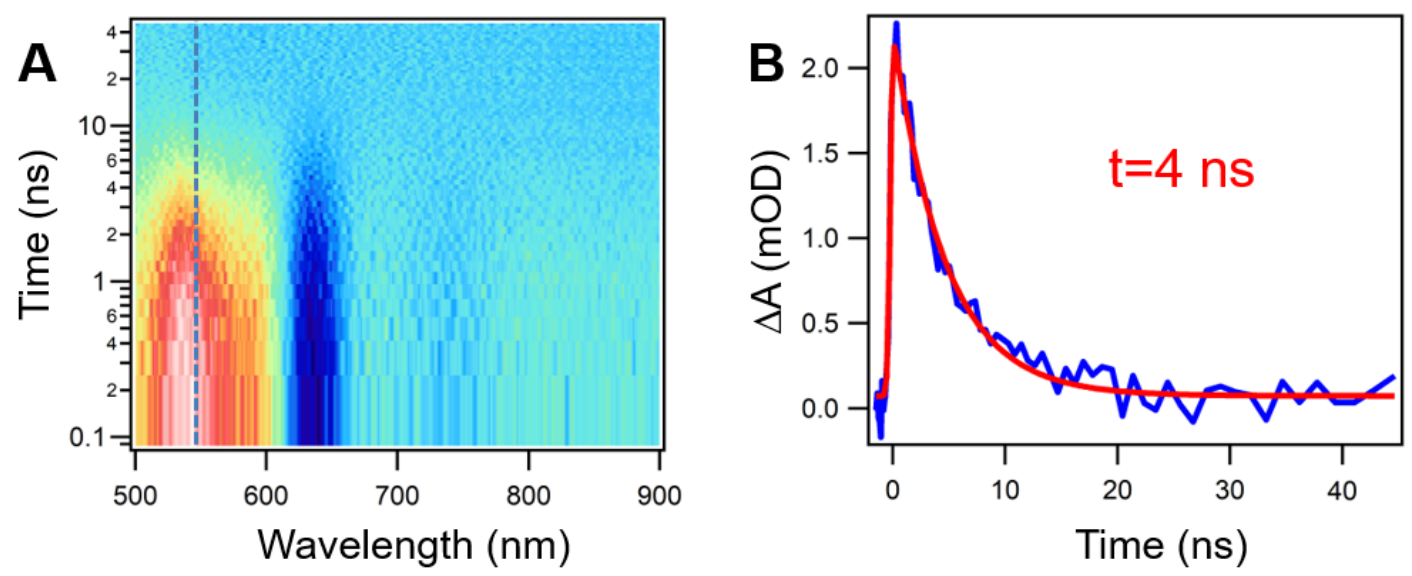

Figure S2. Nanosecond TA measurements on the $\mathrm{Au}_{38}$ nanocluster pumped at $480 \mathrm{~nm}$. (A) $2 \mathrm{D}$ data map of TA between 1 and $45 \mathrm{~ns}$; (B) Decay trace monitored at $550 \mathrm{~nm}$ and the corresponding fit (the lifetime determined to be $4 \mathrm{~ns})$. 
S3. Estimation on the band gap of the Aug9 nanocluster.
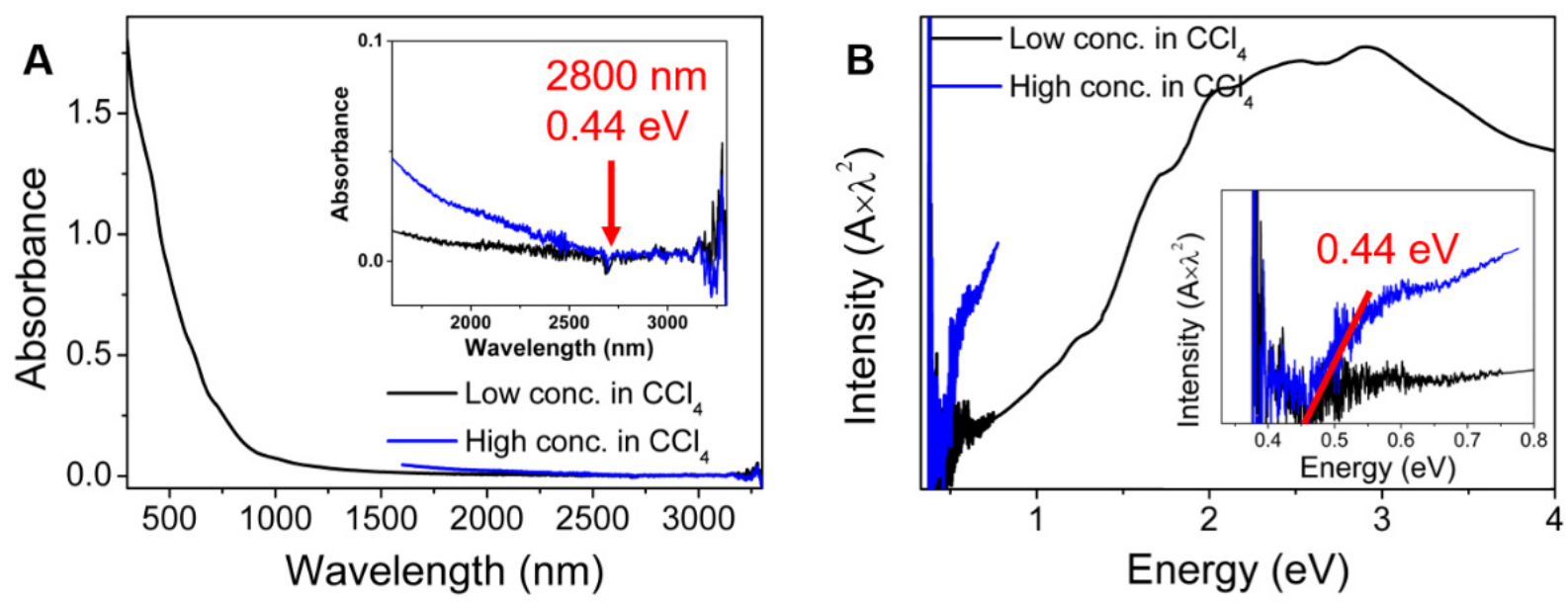

Figure S3. UV-Vis-NIR absorption spectra of $\mathrm{Au}_{99}$ at low and high concentrations. (A) Absorption spectrum on the wavelength scale; (B) absorption spectrum on the photon energy scale. The bandgap is determined to be $0.44 \mathrm{eV}$ by extrapolating the optical absorbance to zero (see insets in both A and B). 


\section{S4. Carrier dynamics of medium sized gold nanoclusters.}

Femtosecond transient absorption (fs-TA) spectroscopic analyses were performed on these three nanoclusters to study their carrier dynamics. Figure S4 A-C show the TA spectra measured between $0.1 \mathrm{ps}$ and $2 \mathrm{~ns}$ after excitation at $370 \mathrm{~nm}(3.3 \mathrm{eV})$. The TA spectra of all three nanoclusters are congested, consisting of broad ESA overlapped with several GSB peaks. One can observe that TA spectra of $\mathrm{Au}_{64}$ show a strong GSB at $525 \mathrm{~nm}$ and two weak GSBs at $650 \mathrm{~nm}$ and $750 \mathrm{~nm}$ (Figure S4A), which agrees with the steady state absorption spectrum. Similarly, in the TA spectra of $\mathrm{Au}_{92}$ and $\mathrm{Au}_{99}$, one can observe three and four GSB peaks, respectively, overlapped with broad ESA band in the visible range. Within the first 5-10 ps, the ESA peaks for all three nanoclusters exhibit a fast decay, which should be explained as the hot carrier cooling to the band edge (Figure S4A-C). In the following $2 \mathrm{~ns}$, there is no significant change in the TA spectra in $\mathrm{Au}_{64}$ (Figure S5), while both ESA and GSB decay to zero for $\mathrm{Au}_{92}$ and $\mathrm{Au}_{99}$ (Figure S4B, C). The slow process should be explained as the electron-hole recombination. It can be seen in Figure $\mathrm{S} 4 \mathrm{D}$ that from $\mathrm{Au}_{64}$ to $\mathrm{Au}_{99}$, the hot carrier cooling becomes slower while the band edge recombination is gradually accelerated. Since the fs-TA signal of $\mathrm{Au}_{64}$ remains unchanged in the measurement window ( $2 \mathrm{~ns}$ ), nanosecond transient absorption measurement was carried out and the lifetime is determined to be $11.5 \mathrm{~ns}$ (see Figure S5 on page s7).
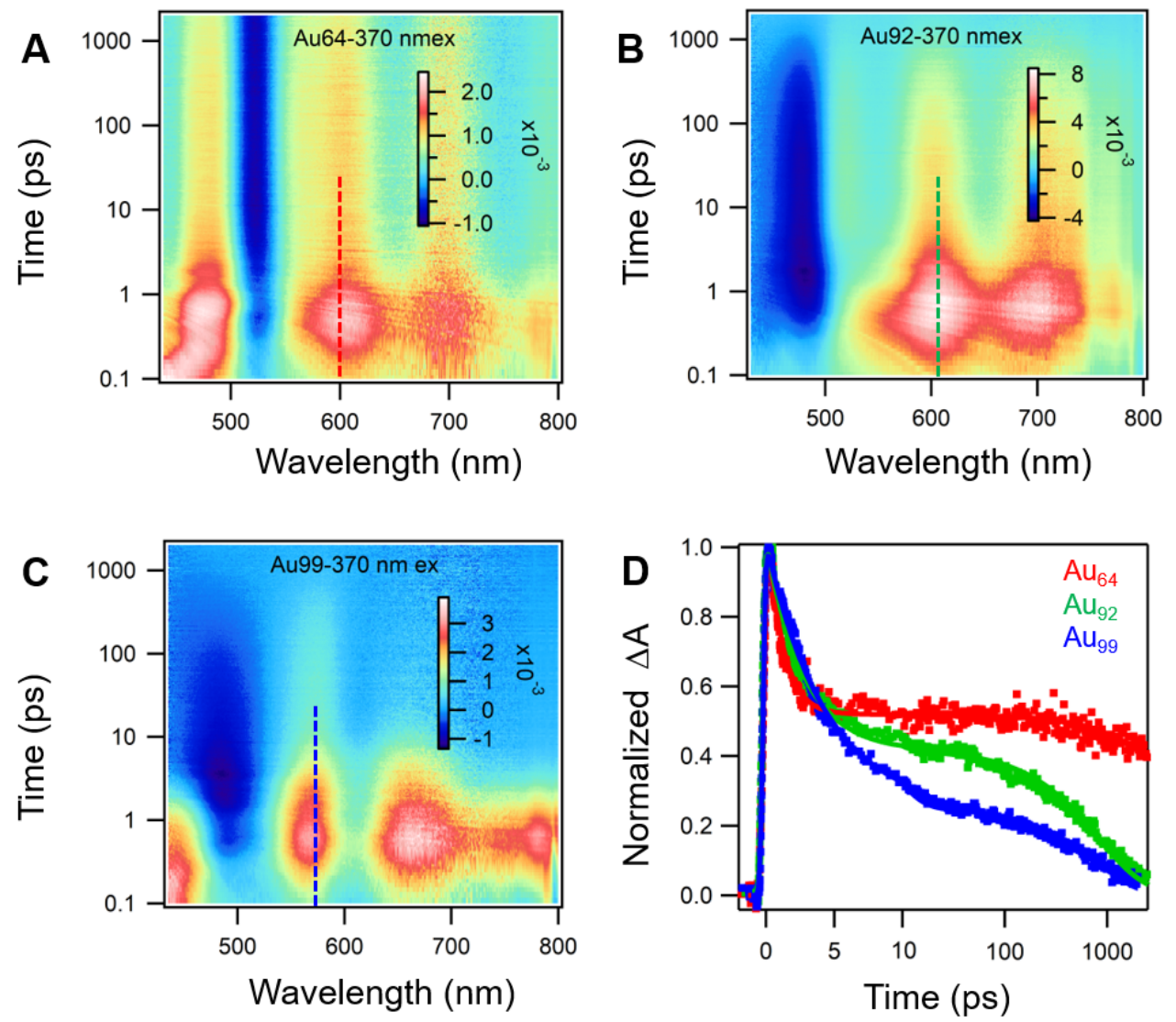

Figure S4. 2D data map of TA spectra of (A) $\mathrm{Au}_{64}$, (B) $\mathrm{Au}_{92}$, and (C) $\mathrm{Au}_{99}$ nanoclusters with excitation at $370 \mathrm{~nm}$ between 0.1 and 2000 picoseconds; (D) Comparison of TA kinetics and the corresponding fits at selected probe wavelengths for three nanoclusters. 
Table S1. Hot carrier and band edge carrier lifetimes of $\mathbf{A u}_{64}, \mathbf{A u}_{92}$ and $\mathrm{Au}_{99}$ nanoclusters pumped at $370 \mathrm{~nm}$.

\begin{tabular}{|l|l|l|l|l|}
\hline & $E_{\mathrm{g}}(\mathrm{eV})$ & $\tau_{\text {band edge }}(\mathrm{ns})$ & $E_{\mathrm{ex}}-E_{\mathrm{g}}(\mathrm{eV})$ & $\tau_{\text {hot carrier }}(\mathrm{ps})$ \\
\hline $\mathrm{Au}_{64}$ & 1.0 & 11.5 & 2.35 & 1.5 \\
\hline $\mathrm{Au}_{92}$ & 0.6 & 1.0 & 2.75 & 2.3 \\
\hline $\mathrm{Au}_{99}$ & 0.44 & 0.7 & 2.91 & 2.8 \\
\hline
\end{tabular}


S5. Nanosecond transient absorption measurements on $\mathrm{Au}_{64}$ nanoclusters.
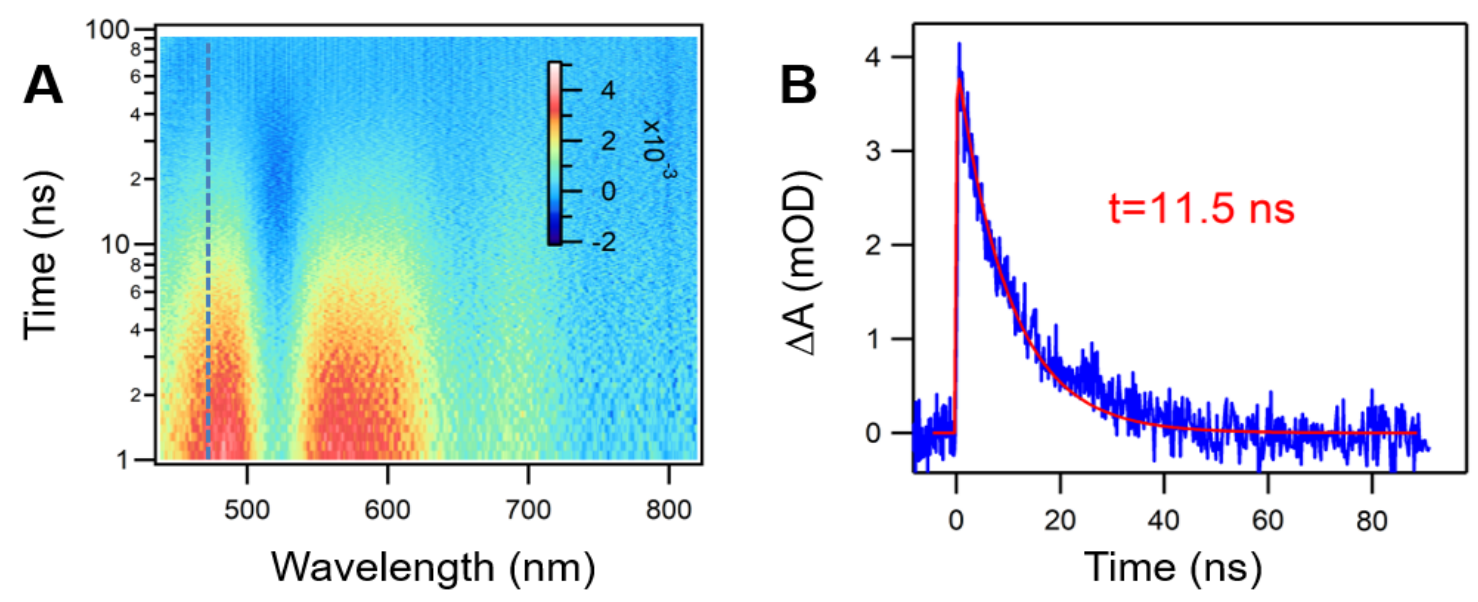

Figure S5. Nanosecond TA measurements on $\mathrm{Au}_{64}$ pumped at $360 \mathrm{~nm}$. (A) 2D data map of TA between 1-100 ns; (B) Decay trace monitored at $480 \mathrm{~nm}$ and the fit (the determined lifetime: $11.5 \mathrm{~ns}$ ). 
S6. Decay associated spectra (DAS) obtained from global fitting of $\mathrm{Au}_{64}, \mathrm{Au}_{92}$ and $\mathrm{Au}_{99}$ nanoclusters.

As shown in Figure S6, two decay components are required to obtain the best fitting quality for TA data pumped at $370 \mathrm{~nm}(3.35 \mathrm{eV})$ for all three samples. From $\mathrm{Au}_{64}$ to $\mathrm{Au}_{99}$, the DAS1 lifetime increases while DAS2 lifetime decreases, which match their band gap evolution. With $1200 \mathrm{~nm}$ excitation (Figure S7), the DAS1 lifetimes for both $\mathrm{Au}_{92}$ and $\mathrm{Au}_{99}$ become shorter.
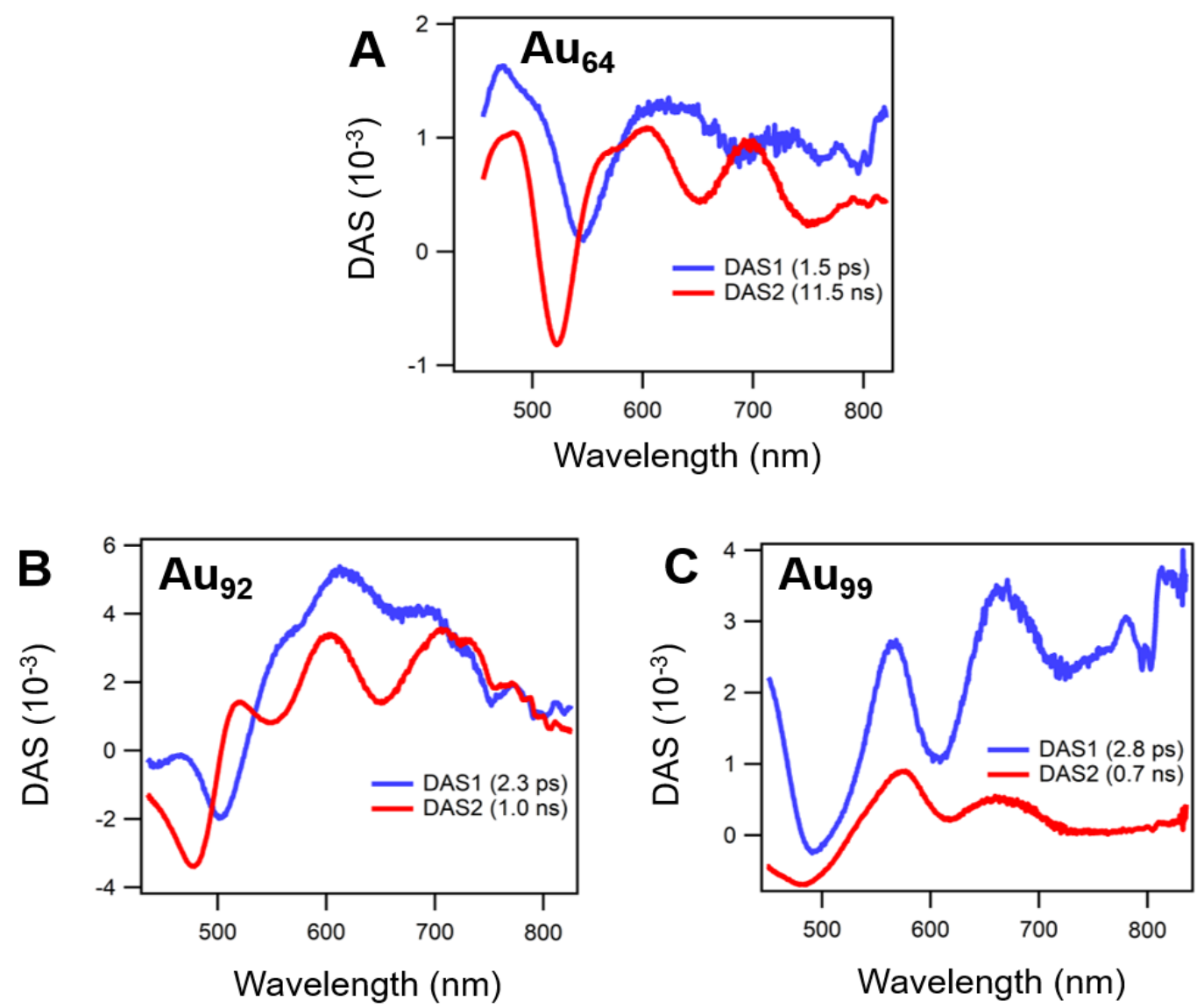

Figure S6. Global fitting results on the TA spectra of $\mathrm{Au}_{64}, \mathrm{Au}_{92}$ and $\mathrm{Au}_{99}$. Decay associated spectra (DAS) obtained from (A) $\mathrm{Au}_{64}$, (B) $\mathrm{Au}_{92}$, and (C) $\mathrm{Au}_{99}$ nanoclusters with excitation of $370 \mathrm{~nm}(3.35 \mathrm{eV})$. 

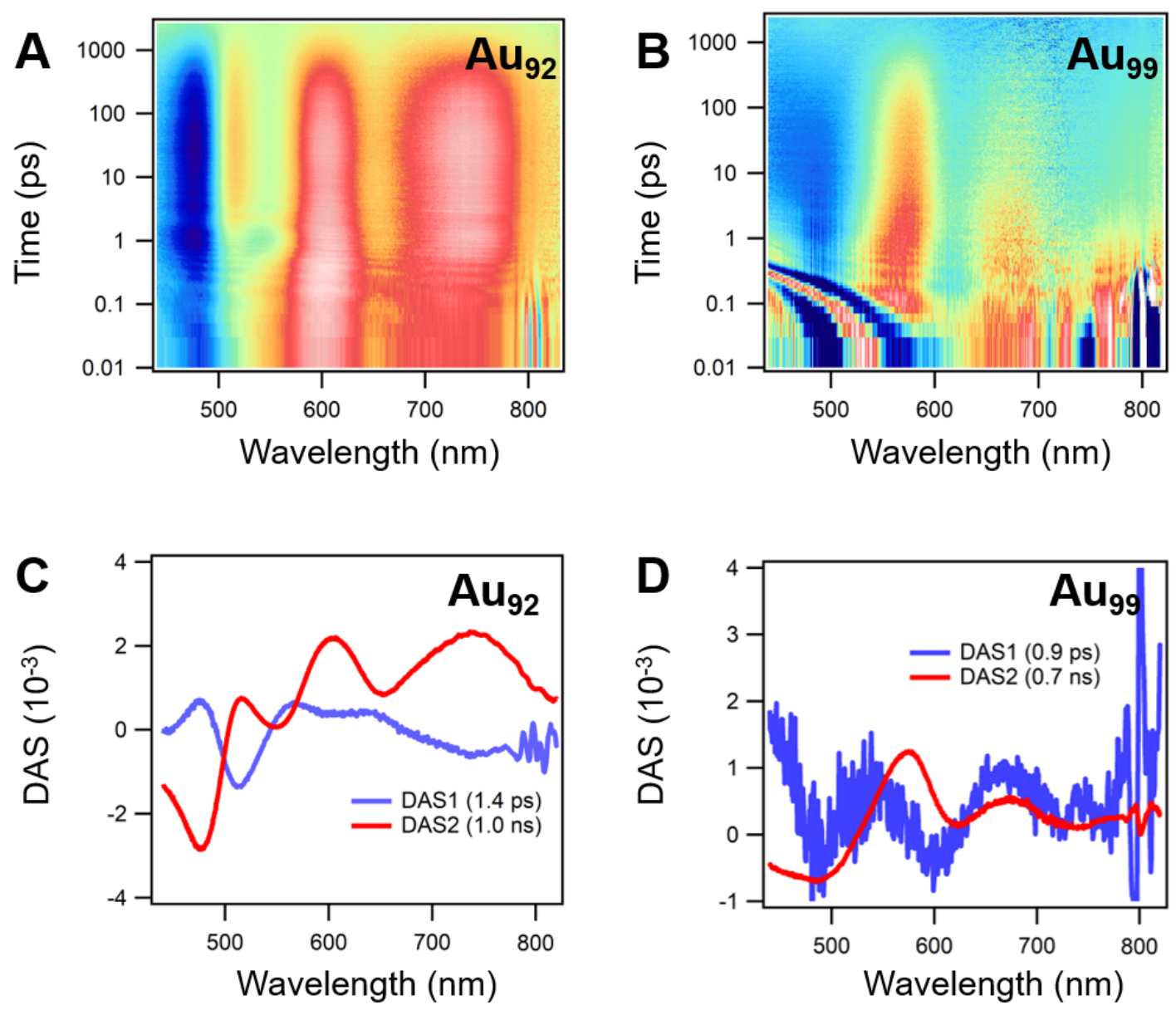

Figure S7. TA measurements on $\mathrm{Au}_{92}$ and $\mathrm{Au}_{99}$ with $1200 \mathrm{~nm}$ excitation. (A-B) TA data map of $\mathrm{Au}_{92}$ and $\mathrm{Au}_{99}$ with $1200 \mathrm{~nm}$ excitation $(1.03 \mathrm{eV}$ ); (C-D) DAS obtained from global fitting of the data. 


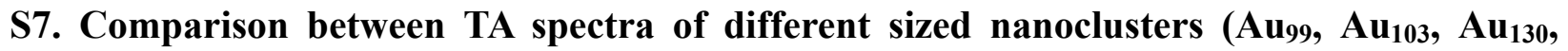
$\left.\mathrm{Au}_{133}, \mathrm{Au}_{144}\right)$
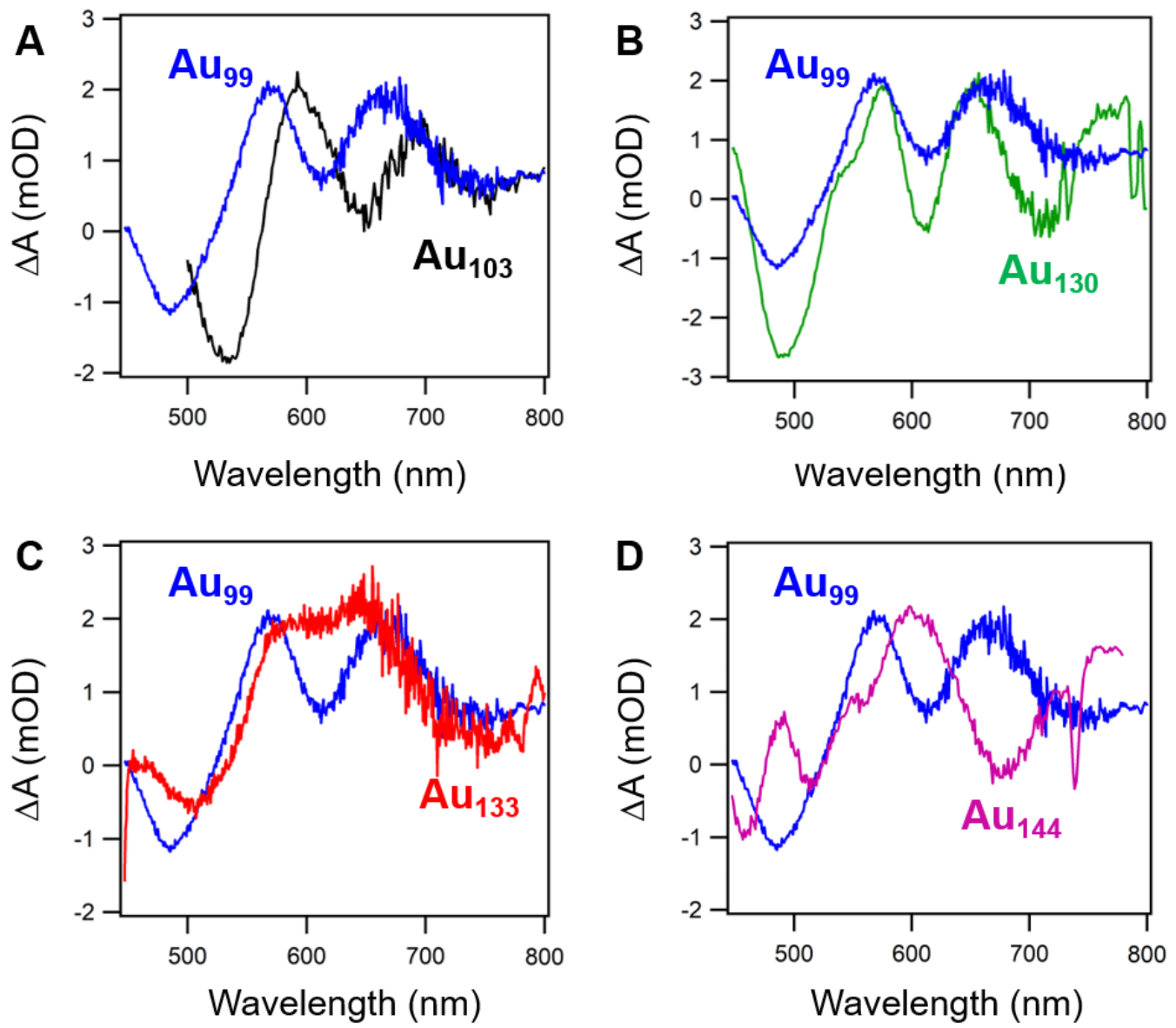

Figure S8. Comparison of TA spectra between $\mathrm{Au}_{99}$ and $(\mathrm{A}) \mathrm{Au}_{103}$, (B) $\mathrm{Au}_{130}$, (C) $\mathrm{Au}_{133}$, and (D) $\mathrm{Au}_{144}$ nanoclusters. It can be found that all these nanoclusters show similar TA profiles. 
S8. Coherent oscillations observed in giant gold nanoclusters $\left(\mathrm{Au}_{99}, \mathrm{Au}_{103}, \mathrm{Au}_{130}, \mathrm{Au}_{133}\right.$, $\left.\mathrm{Au}_{144}, \mathrm{Au}_{246}\right)$
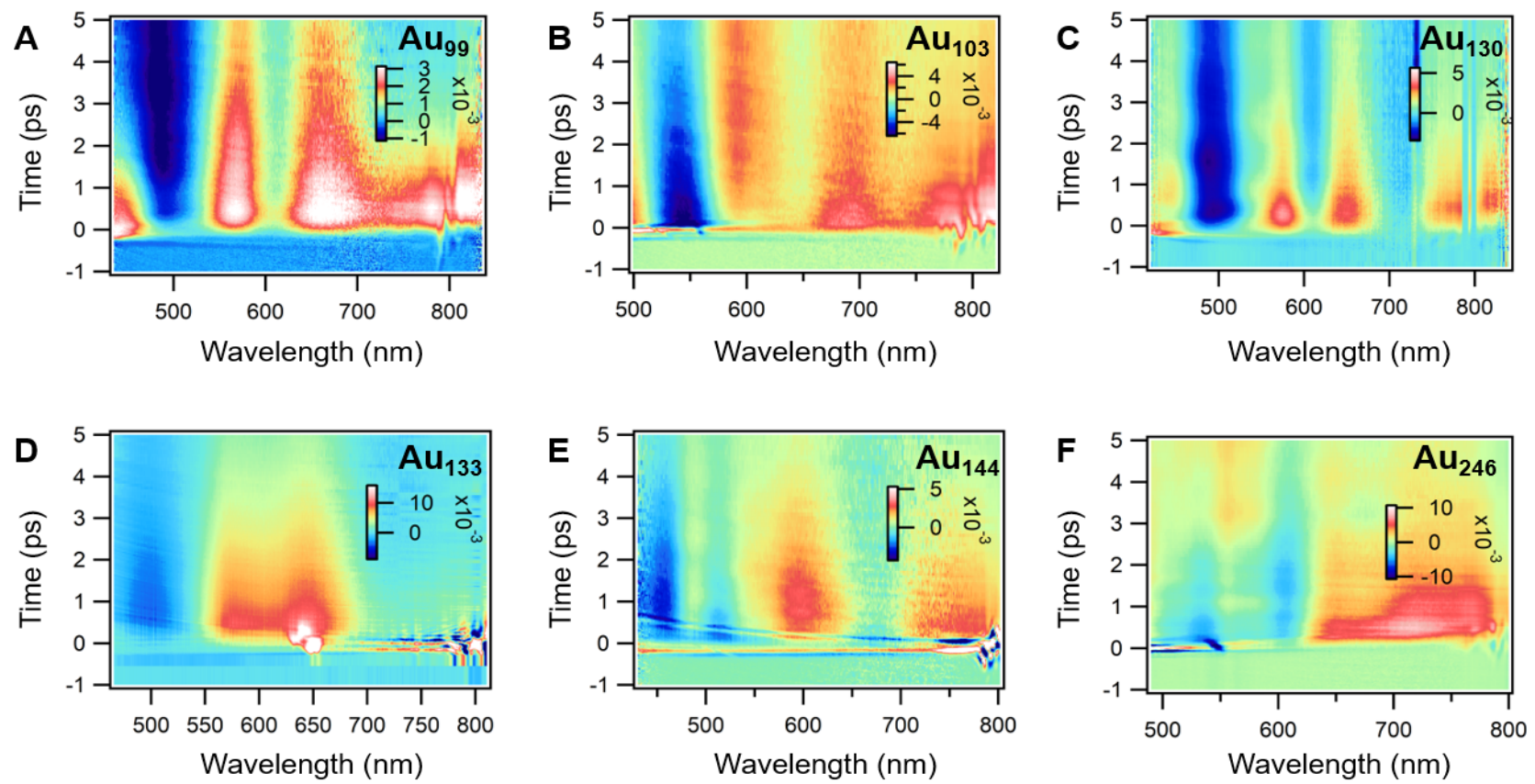

Figure S9. TA data map of $\mathrm{Au}_{99}, \mathrm{Au}_{103}, \mathrm{Au}_{130}, \mathrm{Au}_{133}, \mathrm{Au}_{144}$ and $\mathrm{Au}_{246}$ nanoclusters between -1 and 5 ps.
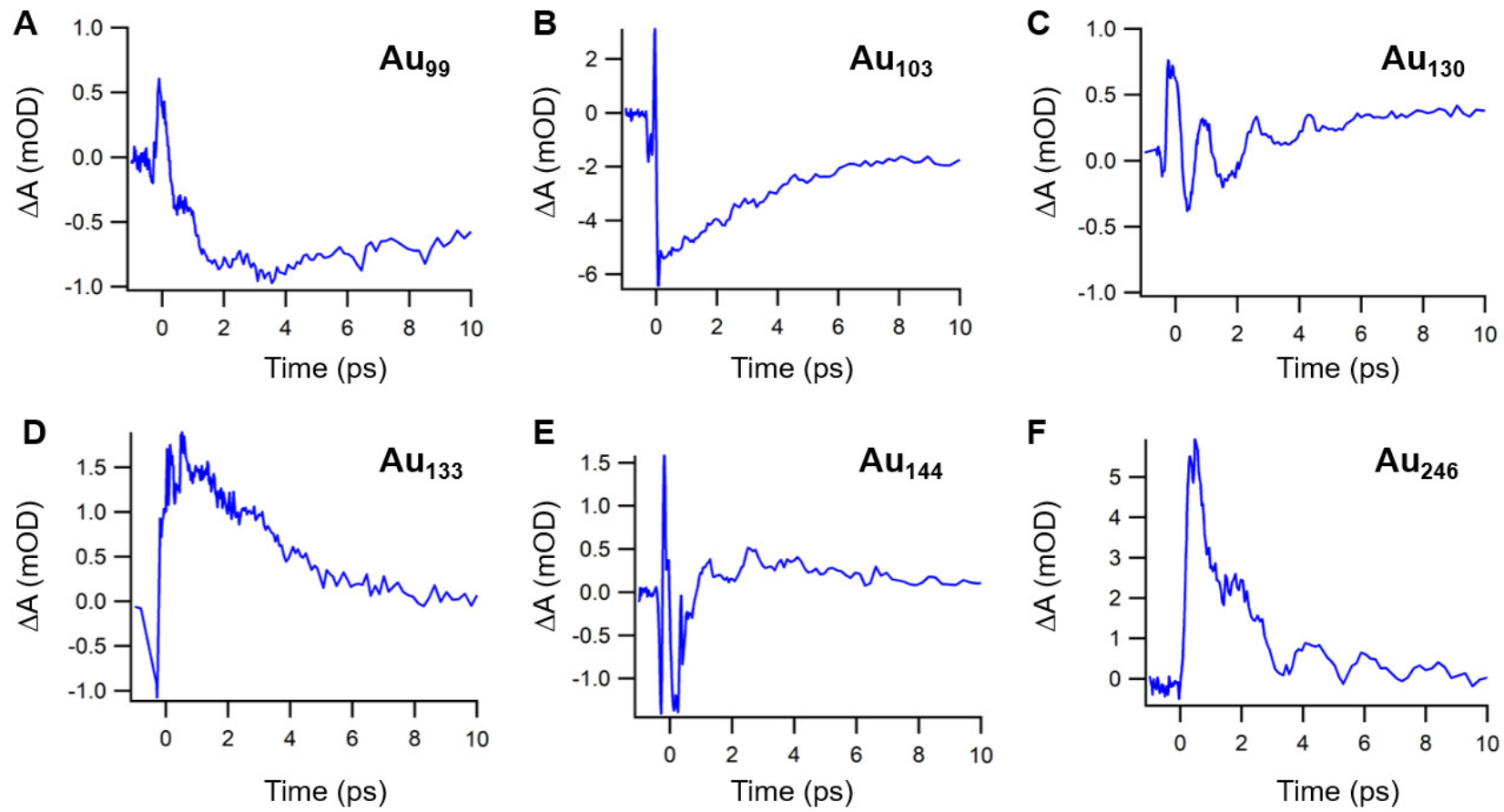

Figure S10. TA decay traces of $\mathrm{Au}_{99}, \mathrm{Au}_{103}, \mathrm{Au}_{130}, \mathrm{Au}_{133}, \mathrm{Au}_{144}$ and $\mathrm{Au}_{246}$ nanoclusters at selected wavelengths. 